RUNNING HEAD: REPLY TO BOWMAN ET AL.

\title{
Reply to Bowman et al: Building the foundations for moving mu suppression research forward
}

\author{
Hannah M. Hobson ${ }^{a}$ \& Dorothy V.M. Bishop ${ }^{b}$
}

\section{a Social, Genetic and Developmental Psychiatry Centre, Institute for Psychiatry, Psychology and Neuroscience, King's College London, SE5 8AF}

\section{${ }^{\mathrm{b}}$ Department of Experimental Psychology, University of Oxford, South Parks Road, OX1 3UD}

It is clear from recent publications that methods of investigating mu suppression, and interpretations of mu suppression findings, are in need of critique, refinement and consensus. To this end, we welcome ongoing debate about the measure, including discussion of our recent registered report. In the report, we evaluated claims that mu suppression represents a valid measure of mirror neuron system (MNS) engagement, and examined how choice of a baseline interval affected mu suppression investigations. The comment by Bowman et al. highlights several current challenges in this field. These include the issue of isolating mirror neuron activity from other cognitive processes that may be reflected in changes in the EEG, understanding variability in individual participants' mu suppression responses, the potential for developmental evidence to aid our interpretations of mu suppression, and finally the shift in the literature from examining "mirror neurons systems" to broader "mirroring". We consider these topics here.

A primary issue is how researchers can extract from suppression what is genuinely neural mirroring, and what is additional activation, driven by non-mirroring processes such as attentional fluctuations. In our report, we argued that if mu suppression was indeed a sufficient MNS measure, then suppression should be greater at central sites for conditions in which participants observed biological stimuli as opposed to non-biological stimuli. Bowman et al. correctly note however that alpha-related suppression due to attentional engagement and mu-related suppression due to sensorimotor processing are not mutually exclusive: observing an action may engage both attention and sensorimotor processes, leading to simultaneous central and occipital suppression. As they 
outline, both in their comment and in their meta-analysis, subtraction can be used to isolate the mirroring components of suppression from those reflecting attentional engagement. Indeed, as Bowman et al. note, this was what our optimal baseline method effectively did, and it was this baseline technique that produced a result in keeping with our specified criteria that central suppression should be greater for biological versus non-biological stimuli. Bowman et al. argue that this shows mu suppression is indeed an adequate measure of neural mirroring (a process the authors distinguish from mirror neuron system engagement - we discuss this distinction later, but use the term neural mirroring here, for clarity), providing experiments are designed and conducted with appropriate baselines (and other important methodological considerations).

However, a few qualifications must be made here, which lead us to remain sceptical where Bowman et al. are optimistic. First, action observation did not always lead to significantly greater suppression than our control stimuli: this significant effect was found only for videos in which hands interacted with objects. Videos of the exact same actions, but without object interaction, did not produce greater mu suppression than videos of kaleidoscope stimuli. Thus, even when an attentional control is used, as in our static image baseline analysis, observing some actions still does not produce the suppression effects expected. Such null findings could of course reveal something of import about the nature of mirroring systems in the brain; however, taken at face value, this finding also curtails the possible roles that neural mirroring may play during action observation. If mu suppression indexes neural mirroring, and significant mirroring only occurs when an action involves interaction with an object (as at least was the case in our study), then neural mirroring appears only to support the processing of transitive actions. An alternative interpretation is that significant suppression findings in mu investigations are not being driven by the processes previously assumed. Indeed, as we discuss in our report, recent investigations suggest that sensory aspects of stimuli may be driving apparent findings of motor mirroring, results that challenge the notion that mu suppression is indexing the activity of a mirror neuron system, classically defined as reflecting primarily motor mirroring ( Coll, Press, Hobson, Catmur, \& Bird, in press.; Coll et al., 2015). In any case, it is a 
simplification to say that action observation will lead to mu suppression, providing that the best methodological controls are employed - suppression does not appear to occur for all action stimuli, a finding that either must restrict the hypothesised role of mirroring processes, or call our measure of them into question..

Another important issue raised by our report is that if mu suppression is a reliable and valid measure of neural mirroring, should we not expect that mu suppression effects will be observed in all typical participants? Indeed, given that some have argued that mu suppression could be harnessed in therapeutic neurofeedback approaches for conditions such as autism spectrum disorder (ASD) (for example, see Pineda, Carrasco, Datko, Pillen, \& Schalles, 2014), it seems imperative to understand the consistency with which the expected mu suppression effects are observed in healthy control groups. Bowman et al. argue that participants from our study who did not show mu suppression could be meaningfully different in some way. And indeed, previous mu suppression investigations in adults have examined variation in mu suppression responses and behavioural characteristics, such as empathy (e.g. Cheng, Yang, Lin, Lee, \& Decety, 2008). In a recent review, we considered whether individual differences in mu suppression in adults could be regarded as a measure of the quality or responsiveness of an individual's MNS (Hobson \& Bishop, 2017). We found that such papers largely failed to correct for multiple comparisons when correlating mu suppression responses with other traits or behaviours, likely leading to false positives. Papers that did correct for multiple comparisons found no associations with the individual differences purported to be related to MNS functioning (Silas, Levy, Nielsen, Slade, \& Holmes, 2010). Furthermore, such work has failed to provide any evidence for the test-retest reliability of mu suppression as an individual differences measure (a crucial step before relating differences in mu suppression to other stable participant characteristics). We would thus be cautious about claiming that this group of non-suppressors are meaningfully different, at least until the reliability of mu suppression can be established; if mu suppression is not reliably found in many people, then it is premature to use it as a target for neurofeedback in clinical populations. 
In our recent review, we did not consider the mu suppression investigations conducted with children, but Bowman et al. note this is a burgeoning literature, and that developmental approaches could be used to investigate whether mu suppression is reflective of mirroring processes. Studying children with different degrees of motor and social development does indeed present an interesting and informative approach to unpicking mu suppression's nature. However, thus far developmental investigations have used mu suppression to test developmental theories, rather than using developmental approaches to validate mu suppression, as Bowman et al. suggest. Furthermore, we consider the present developmental mu suppression evidence to be rather mixed. For instance, the associations reported by Bowman et al. (Bowman, Thorpe, Cannon, \& Fox, 2016) were based on mu suppression during action execution only, not suppression from action observation, as significant mu suppression during observation was not achieved. This is in keeping with our registered report on adults, in which strong suppression was seen for participants' own movements, but much more limited evidence of suppression was found at the central regions during the observation conditions. Across the developmental mu suppression literature, each paper seems to have used a different measure of infant motor or action ability, and usually there are several of them. For example, Cannon et al. (2016) found only one of four of their grasp measures predicted mu suppression, and none of their grasp-reach measures predicted suppression during execution, which again would seem to suggest some key differences in mu suppression during observation vs. execution. Together, we argue that current developmental work paints a picture that is not wholly how we would predict a measure of neural mirroring to look. Following on from this pioneering work on infant mu suppression, we suggest that preregistered approaches, based on this initial body of work, would be beneficial here to bolster claims that mu suppression in infants in meaningfully related to their development of motor skill or action understanding.

Mirror neurons were originally described nearly 30 years ago (Rizzolatti et al., 1988), and the theoretical accounts of the MNS have of course evolved over time. Bowman et al. suggest that researchers need not be concerned with whether mu suppression is reflecting a mirror neuron 
system synonymous with the original animal work. This comment reflects a move away from talking about mirror neuron systems to a rather general property of "neural mirroring", and away from cells in specific brain regions to wider brain networks. This progression may well reflect reality; there is no reason to assume that mirror neurons are restricted to the homologue of the monkey $\mathrm{F} 5$ region, and indeed sequential fMRI-EEG studies suggest that mu suppression is likely to reflect the activity of a number of areas (including areas considered part of the MNS and non-MNS areas) (Braadbaart, Williams, \& Waiter, 2013). However, insofar as mu suppression reflects the activity of a broader network, the issue of what it truly captures comes more into focus. Is it sufficient to say that when suppression is observed during action-observation, even with proper baseline conditions, that this suppression, arising from the activity of a broad network of regions, reflects simply "mirroring"? Describing it this way misses the point that if the sum of the activity of these brain regions even does amount to a process we might consider to be mirroring, there are clearly many sub-processes involved. Suppression considered to be reflective of "mirroring" could also incorporate processes of action preparation, or simple associations between stimuli and motor activity (the demonstration of mu suppression to sheet music makes a strong case for this associative account; see Behmer \& Jantzen, 2011). Furthermore, as noted earlier, recent investigations have suggested the mirroring component of mu suppression may be limited to sensory elements, and that motor mirroring is not reflected in mu suppression (Coll, Bird, Catmur, \& Press, 2015; Coll et al., in press). Clearly, if the field is to move away from examining mirror neuron systems and into investigations of broader neural mirroring, this construct will need to be properly defined, and whether mu suppression truly captures the whole part of it, or merely some sub-construct, or a related sensorimotor process that is not what we could consider mirroring, will need to be examined. We urge future researchers to consider: what, precisely, is mirroring, and is it a construct with psychological utility?

Finally, we would dispute the claim by Bowman et al. that the widespread mu suppression effects in their meta-analysis suggest mu suppression is a robust effect. This misses the point that an effect found under so many conditions and set-ups, some of which have been shown now by our 
registered report to be problematic, is unlikely to be pure or valid across these studies. In a recent article, Luck and Gaspelin (2017) noted the ease with which 'significant' results could be obtained in studies of event-related potentials (ERPs), because of the numerous points in the analysis where there was flexibility in how data were treated, the tendency to use 'double dipping', i.e. selecting data for analysis only after observing which portions showed the effect of interest, and use of exploratory multiway analysis of variance without correction for multiple testing. All of these criticisms apply to the mu suppression literature, with the addition that not only time intervals but also frequency bands are often selected for analysis post hoc. Viewed in this light, the fact that studies that look for mu suppression usually find some consistent evidence is far from compelling. We suggest that journals in this field need to move to require either preregistration of hypotheses, or independent replication of findings, if it is to progress.

How best, then, to move forward? We agree with Bowman et al. that there are numerous ways in which mu suppression studies might be improved. We are not calling for researchers to abandon the study of mu suppression, but rather to do more basic research to establish the validity and reliability of mu suppression a mirror neuron measure. At this time, we take a more sceptical view than Bowman et al. of the reproducibility of much of the existing literature on this topic.

\section{Acknowledgements}

During the original Registered Report, Hannah Hobson was supported by the Medical Research Council, through a Medical Sciences Division studentship, MRC grant 1242092. Dorothy Bishop is supported by Wellcome Trust programme grant 082498/Z/07/Z.

\section{References}

Behmer, L. P., \& Jantzen, K. J. (2011). Reading sheet music facilitates sensorimotor mudesynchronization in musicians. Clinical Neurophysiology, 122(7), 1342-7. http://doi.org/10.1016/j.clinph.2010.12.035 
Bowman, L. C., Thorpe, S. G., Cannon, E. N., \& Fox, N. A. (2016). Action mechanisms for social cognition: Behavioral and neural correlates of developing Theory of Mind. Developmental Science, 1-16. http://doi.org/10.1111/desc.12447

Braadbaart, L., Williams, J. H. G., \& Waiter, G. D. (2013). Do mirror neuron areas mediate mu rhythm suppression during imitation and action observation? International Journal of Psychophysiology, 89(1), 99-105. Retrieved from http://ovidsp.ovid.com/ovidweb.cgi?T=JS\&PAGE=reference\&D=psyc8\&NEWS=N\&AN=201322916-001

Cannon, E. N., Simpson, E. A., Fox, N. A., Vanderwert, R. E., Woodward, A. L., \& Ferrari, P. F. (2016). Relations between infants' emerging reach-grasp competence and event-related desynchronization in EEG. Developmental Science, 19(1), 50-62. http://doi.org/10.1111/desc.12295

Cheng, Y., Yang, C.-Y., Lin, C.-P., Lee, P.-L., \& Decety, J. (2008). The perception of pain in others suppresses somatosensory oscillations: a magnetoencephalography study. Neurolmage, 40(4), 1833-40. http://doi.org/10.1016/j.neuroimage.2008.01.064

Coll, M.-P., Bird, G., Catmur, C., \& Press, C. (2015). Cross-modal repetition effects in the mu rhythm indicate tactile mirroring during action observation. Cortex, 63, 121-131. http://doi.org/10.1016/j.cortex.2014.08.024

Coll, M.-P., Press, C., Hobson, H., Catmur, C., \& Bird, G. (in press). Crossmodal classification of mu rhythm activity during action observation and execution suggests specificity to somatosensory features of actions. The Journal of Neuroscience.

Coll, M., City, Q., Coll, M., Bird, G., Catmur, C., Press, C., ... Centre, D. P. (2015). Crossmodal repetition effects in the mu rhythm indicate tactile mirroring during action observation. Cortex, $63,121-131$. 
Hobson, H. M., \& Bishop, D. V. M. (2017). The interpretation of mu suppression as an index of mirror neuron activity : past , present and future. Royal Society Open Science, 4(160662). http://doi.org/10.1098/rsos.160662

Luck, S. J., \& Gaspelin, N. (2017). How to get statistically significant effects in any ERP experiment ( and why you shouldn' t ). Psychophysiology, 54, 146-157. http://doi.org/10.1111/psyp.12639

Pineda, J. A., Carrasco, K., Datko, M., Pillen, S., \& Schalles, M. (2014). Neurofeedback training produces normalization in behavioural and electrophysiological measures of high-functioning autism. Philosophical Transactions of the Royal Society of London. Series B, Biological Sciences, 369, 20130183.

Rizzolatti, G., Camarda, R., Fogassi, L., Gentilucci, M., Luppino, G., \& Matelli, M. (1988). Functional organization of inferior area 6 II Area F5 and the control of distal movements. Experimental Brain Research, 71, 491-507. http://doi.org/10.1007/BF00248742

Silas, J., Levy, J. P., Nielsen, M. K., Slade, L., \& Holmes, A. (2010). Sex and individual differences in induced and evoked EEG measures of action observation. Neuropsychologia, 48(9), 2417-26. http://doi.org/10.1016/j.neuropsychologia.2010.03.004 